\title{
Prodromal schizophrenia in primary care: a randomised sensitisation study
}

\author{
Andor E Simon, Sabrina Jegerlehner, Thomas Müller, Katja Cattapan-Ludewig, \\ Peter Frey, Marcus Grossenbacher, Erich Seifritz and Daniel Umbricht
}

\begin{abstract}
Background

GPs are often the first point of contact for patients with prodromal schizophrenia. Early intervention, and therefore early detection, of schizophrenia is pivotal for the further disease course. However, recent studies have revealed that, due to its low prevalence in general practice and its insidious features, prodromal schizophrenia often remains unnoticed.
\end{abstract}

\section{Aim}

To test whether a repeated sensitisation method using clinical vignettes can improve diagnostic knowledge of GPs.

\section{Design of study}

Postal survey using anonymous questionnaires. Repeated sensitisation model using clinical vignettes.

\section{Setting}

GPs in three distinct regions in Switzerland covering a general population of 1.43 million.

\section{Method}

The study was conducted between September 2008 and October 2009. Questionnaires were sent to 1138 GPs at baseline, and at 6 and 12 months. After randomisation, $591 \mathrm{GPs}$ were sensitised at 1, 3, and 5 months, while no sensitisation was carried out in the remaining 547 GPs.

\section{Results}

The overall response rate was $66 \%$ (750 GPs). Sensitised GPs demonstrated a highly significant increase in diagnostic knowledge at 6 and at 12 months when compared to their own baseline knowledge scores and also to non-sensitised GPs $(P<0.001)$. In particular, awareness of insidious features, such as functional decline and social withdrawal as signs of prodromal schizophrenia, accounted for this effect.

\section{Conclusion}

Theoretical knowledge of prodromal schizophrenia among GPs can successfully be increased by repeated sensitisation models using clinical vignettes.

\section{Keywords}

family practice; mental health; psychosis, early; schizophrenia, prodromal.

\section{INTRODUCTION}

Schizophrenia is among the most severe and disabling mental disorders. Reducing the duration of untreated psychosis after the onset of a first psychotic episode has been associated with a more favourable course of the disease., ${ }^{1,2}$ In recent years, efforts have focused on intervening even as early as the prodromal phase preceding the first onset of schizophrenia. However, most prodromal symptoms remain non-specific, and it is not until the late prodromal phase that attenuated psychotic-like or even brief limited psychotic symptoms emerge. ${ }^{3,4}$ Among the earliest signs of schizophrenia, insidious features, such as decline of social functioning and early social withdrawal, have often been reported. ${ }^{5}$

AE Simon, MD, senior lecturer and psychiatrist, University Hospital of Psychiatry, University of Bern and Specialised Early Psychosis Outpatient Service for Adolescents and Young Adults, Department of Psychiatry, Bruderholz, Switzerland. S Jegerlehner, medical student and research fellow; T Müller, $M D$, chief psychiatrist, University Hospital of Psychiatry; $\boldsymbol{P}$ Frey, $M D$, research director, Bern Institute of General Practice, University of Bern, Bern, Switzerland. K Cattapan-Ludewig, $M D$, chief psychiatrist, University Hospital of Psychiatry, University of Bern and Sanatorium Kilchberg, Psychiatric Clinic, Kilchberg, Switzerland. M Grossenbacher, MD, family physician and president of Family Physicians Association, Bern, Switzerland. E Seifritz, MD, chief psychiatrist, University Hospital of Psychiatry, University of Zurich, Zurich, Switzerland. D Umbricht, MD, senior lecturer and research psychiatrist, Clinical Research and Exploratory Medicine Neuroscience, Hoffmann-La Roche Ltd., Basel, Switzerland.

Address for correspondence

Andor E Simon, Specialised Early Psychosis Outpatient Service for Adolescents and Young Adults, Psychiatric Outpatient Services, Department of Psychiatry, 4101 Bruderholz, Switzerland. E-mail: andor.simon@bluewin.ch

Submitted: 25 January 2010; Editor's response: 22 February 2010; final acceptance: 30 March 2010.

(C)British Journal of General Practice

This is the full-length article of an abridged version published in print. Cite this article as: Br J Gen Pract 2010; DOI: 10.3399/bjgp10X515377. 
Studies investigating help-seeking pathways have shown that these individuals often seek initial help from their family physicians. ${ }^{6-9}$ This finding points towards the need to deliver information to GPs about early warning signs of schizophrenia. However, as is commonly the case for mental health problems, patients may not instantly reveal their psychological difficulties. ${ }^{10-12}$ Furthermore, the insidious features of prodromal schizophrenia often remain underidentified in primary care. ${ }^{13,14}$ In the first comprehensive study to assess the ability of Swiss GPs $(n=1089)$ to detect prodromal schizophrenia, GPs displayed good skills in detecting the more prominent features, such as hallucinations or bizarre behaviour, but under-identified features, such as functional decline or social withdrawal. ${ }^{13}$ These results were replicated in an international GP study conducted in seven additional countries in Europe, Northern America, and Oceania between 2003 and 2005 among 2784 GPs. ${ }^{14}$

Against this background, the ideal sensitisation model needs to meet the following cardinal criteria: sensitisation must be short, clear, easily manageable, and of high clinical relevance. As there is strong evidence that disease-specific awareness of GPs is not increased by unimodal continuing medical education, sensitisation needs to be multimodal and repetitious. ${ }^{15,16}$

This article presents the results of a randomised study among 1138 GPs from three distinct regions in Switzerland. It assessed whether a sensitisation model meeting the above criteria and using repeated clinical vignettes could contribute to increased diagnostic knowledge of the core symptoms of prodromal schizophrenia. It was hypothesised that sensitised GPs, as compared to non-sensitised GPs, would show an increased awareness of these core symptoms from baseline (Q1) to a 6-month sensitisation period (Q2). The study explored whether a sustained effect of the sensitisation was still present at 12 months (Q3).

To date, only a few studies have been conducted to assess the effect of education models in primary care in relation to prodromal schizophrenia. These studies focused on first-episode patients and did not assess the effect on diagnostic knowledge in GPs. ${ }^{9,17}$ The present study is the first on the initial prodromal phase of schizophrenia to assess the effect of a sensitisation model in primary care, and it is also the first study in the entire field of early psychosis to apply this particular sensitisation model.

\section{METHOD}

\section{Participants}

The study was conducted among all GPs in three distinct regions in Switzerland: the canton of Bern

\section{How this fits in}

The majority of patients with prodromal schizophrenia initially contact their GP.

The symptoms of prodromal schizophrenia, however, are insidious and not specific, and thus may remain undetected. Multimodal sensitisation using repeated clinical vignettes and ad hoc auto-examination led to a sustained increase in GPs' diagnostic knowledge of core symptoms of prodromal schizophrenia $(P<0.001)$. Such sensitisation models may be important elements for psychiatrists to assist GPs in their challenging task of detecting patients with prodromal schizophrenia.

(744 GPs; population 960 000), the canton of BaselLandschaft (254 GPs; population 300000 ), and a part of the canton of Zurich (188 GPs; population 170 000). Complete addresses of GPs were obtained by the three GP associations of the respective study regions. The presidents of all three GP associations were included in the development of the study design. Approval from local ethics committees was not required.

The study design is illustrated in Figure 1. Fifteen GPs were identified as practising in general hospital settings and thus were excluded from the targeted GP sample. Prior to the study, the baseline GP sample $(n=1171)$ was randomised into two samples by alternately assigning every second address to the sensitised GP group (GPsens; $n=607$ ) and the nonsensitised GP group (GPnsens; $n=564$ ) respectively. At baseline, all GPs received an information letter by regular mail about the study design. Of the 1171 GPs, 33 had meanwhile retired from their GP activity. Thus the corrected GP sample at baseline was 1138 (GPsens: $n=591$; GPnsens: $n=547$ ).

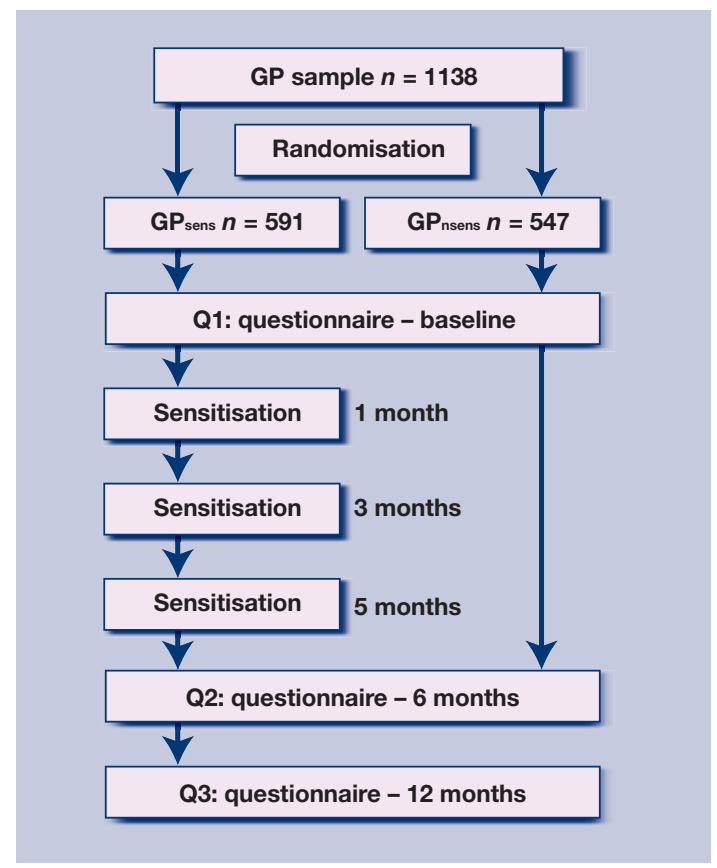




\section{Questionnaire}

At three time points (Q1-Q3), all GPs received a short postal questionnaire. The questionnaires were anonymous and written in German. A coding system (the day and the year of birth of their mother, the day of birth of their father, as well as their own sex) was used to enable matching of GPs who responded to more than one mailing. This coding system ensured anonymity and was initially developed for the former Swiss GP study. ${ }^{13}$ It was approved by the Eidgenössisches Büro für Datenschutz (Federal Office of Data Protection).

The core question was a multi-item question to examine which symptoms are assessed by GPs and which additional information they include to corroborate the diagnosis of a suspected prodromal schizophrenia (Appendix 1).

\section{Core items}

In the multi-item question of the questionnaires, GPs were asked to indicate exactly five core items out of a selection of 20: decline in social functioning, social withdrawal, family history, information from significant others, and delusion-like ideas. In accordance with the methods applied previously in the Swiss and international GP studies, ${ }^{13,14}$ these five core items were selected as they represent classic warning signs as well as factors associated with an increased risk of psychosis, and can be easily assessed by GPs. Specifically, functional decline and social withdrawal have both frequently been reported in the early stages of schizophrenia, ${ }^{5}$ with a recommendation to obtain information from significant others because patients in the early stages often 'seal over'; that is, they deny both social withdrawal and functional decline..$^{18}$ Delusion-like ideas have recently been shown to have a high predictive value for later transition to psychosis in at-risk patients, in particular in association with functional decline and social withdrawal. ${ }^{19}$ Finally, a positive family history was chosen because of its recognised association with an increased risk for schizophrenia. ${ }^{20}$

\section{Intervention}

Letters, each containing two clinical vignettes (see example, Appendix 1), were sent to sensitised GPs (GPsens) at 1, 3, and 5 months. Clinical vignettes differed at each time point. Non-sensitised GPs (GPnsens) did not receive any mailing. The clinical vignettes were very brief and required GPs to spend no more than 1 to 2 minutes on them. The vignettes described clinical situations in which GPs were asked to indicate how they would assess the risk for prodromal schizophrenia. Together with the vignettes, they were provided with the same 20 items with potential symptoms and assessment procedures as in the multi-item question of the questionnaires, again with exactly five to choose from. On the reverse side of the letter, a briefly formulated solution to the vignettes was provided, and the five correct items were indicated on the list of potential symptoms and assessment procedures. No further sensitisation occurred between Q2 and Q3.

\section{Outcome measure}

The principal outcome measure was diagnostic knowledge, as measured by a composite score (cscore) of the core items. The correct identification for any one of these items was rated with a score of 2; thus if all five items were correctly identified, the c-score was 10 . This ranking model was applied to compare the results with those obtained in the authors' previous studies on Swiss GPs, ${ }^{13}$ and an international group of GPs. ${ }^{14}$ In these latter studies, further scores were calculated using the remaining items that appear in the multi-item question of the questionnaire. As the study aimed to sensitise GPs towards recognising the five core items, the other items were not included in the analysis of the present study.

\section{Analysis}

Data were analysed using SPSS (version 13.0). Continuous variables were compared with $t$-tests or analyses of variance (ANOVA), and categorical variables with $\chi^{2}$ tests. An $\alpha$ level of 0.05 was considered significant. The c-scores were compared between GPsens and GPnsens at all time points (Q1-Q3). In a primary approach, all returned

Table 1. Mean c-scores of GPs.

\begin{tabular}{|c|c|c|c|c|c|c|c|c|c|c|c|c|c|c|c|}
\hline & \multicolumn{5}{|c|}{ All responses included ${ }^{a}$} & \multicolumn{5}{|c|}{$\leq 5$ responses included ${ }^{a}$} & \multicolumn{5}{|c|}{ Answers in Q1 to Q3 } \\
\hline & \multicolumn{2}{|c|}{ GP nsens } & \multicolumn{3}{|c|}{ GP sens } & \multicolumn{2}{|c|}{$\mathrm{GP}_{\text {nsens }}$} & \multicolumn{2}{|c|}{$G P_{\text {sens }}$} & \multirow[b]{2}{*}{$P$-value ${ }^{c}$} & \multicolumn{2}{|c|}{$\mathrm{GP}_{\text {nsens }}$} & \multicolumn{2}{|c|}{ GP sens } & \multirow[b]{2}{*}{$P$-value ${ }^{c}$} \\
\hline & $n$ & $\mathrm{c}^{\mathrm{b}}$ & $n$ & c & $P$-value ${ }^{c}$ & $n$ & c & $n$ & c & & $n$ & $\mathrm{c}$ & $n$ & c & \\
\hline Q1 & 211 & 5.12 & 234 & 5.29 & 0.435 & 160 & 4.23 & 173 & 4.35 & 0.547 & 37 & 5.41 & 56 & 5.40 & 0.977 \\
\hline Q2 & 149 & 5.63 & 176 & 6.64 & $<0.001$ & 99 & 4.73 & 143 & 6.28 & $<0.001$ & 37 & 5.89 & 56 & 6.96 & 0.019 \\
\hline Q3 & 146 & 5.53 & 174 & 6.06 & 0.046 & 100 & 4.52 & 145 & 5.67 & $<0.001$ & 37 & 5.35 & 56 & 6.21 & 0.045 \\
\hline
\end{tabular}

${ }^{a}$ Responses in question 3 of the survey. ${ }^{b} \mathrm{C}=$ composite score. ${ }^{\mathrm{ct}}$-tests. Q1 = baseline questionnaire. Q2 = questionnaire at 6 months. Q3 = questionnaire at 12 months. 
questionnaires were included without any restrictions on the amount of answers given in the multi-item question. Ratios of GPs that gave a maximum of five answers in the multi-item question were: Q1 = $70.8 \%, \mathrm{Q} 2=72.9 \%$, and $\mathrm{Q} 3=76.6 \%$. As the likelihood of correct answers theoretically increases with an increasing number of answers given, the study further compared GPsens and GPnsens who had only given a maximum of five answers as requested in the sensitisation model. Finally, those GPs who had responded in all three surveys were compared according to the study coding system. For the latter groups, repeated measures ANOVAs and post hoc ANOVAs were further used to calculate time $\times$ group interaction on c-scores.

\section{RESULTS}

Overall, a total of $750(66 \%)$ of all contacted GPs responded at least once to the study surveys. Response rates were: Q1 39\% (40\% GPsens and 38\% GPnsens); Q2 29\% (32\% GPsens and 27\% GPnsens); Q3 28\% (29\% GPsens and $27 \%$ GPnsens). All $\chi^{2}$ tests comparing response rates between GPsens and GPnsens were non-significant. Table 1 shows mean c-scores with comparisons between GPsens and GPnsens for Q1-Q3. At Q2, GP groups significantly differed in all three sampling approaches. When only those questionnaires with a maximum of five answers in the multi-item question were included, this difference was still highly significant at Q3.

According to the study coding system, a total of 93 GPs (56 GPsens and 37 GPnsens) responded in all three surveys. There was a trend for significance in the time $\times$ group interaction on c-scores between GPsens and GPnsens $\left(F_{(2,182)}=2.562, P=0.080\right)$. A highly significant time effect was found for GPsens $\left(F_{(2,110)}=12.403, P<0.001\right)$, but not for GPnsens $\left(F_{(2,72)}\right.$ $=1.086, P=0.343$; Figure 2).

Figures 3 and 4 show how often both GP groups identified the five core items that were highlighted during sensitisations when only a maximum of five answers were permitted. While only a modest increase was observed in GPnsens in one single item (family history of psychosis), GPsens showed a large increase in recognising the insidious features of prodromal schizophrenia, such as social withdrawal and decline in social functioning, and in emphasising information from significant others as an important assessment tool. GPsens identified delusion-like or unusual thoughts slightly less often in Q3 than in the previous surveys.

\section{DISCUSSION}

\section{Summary of main findings}

The study hypothesis was robustly confirmed:

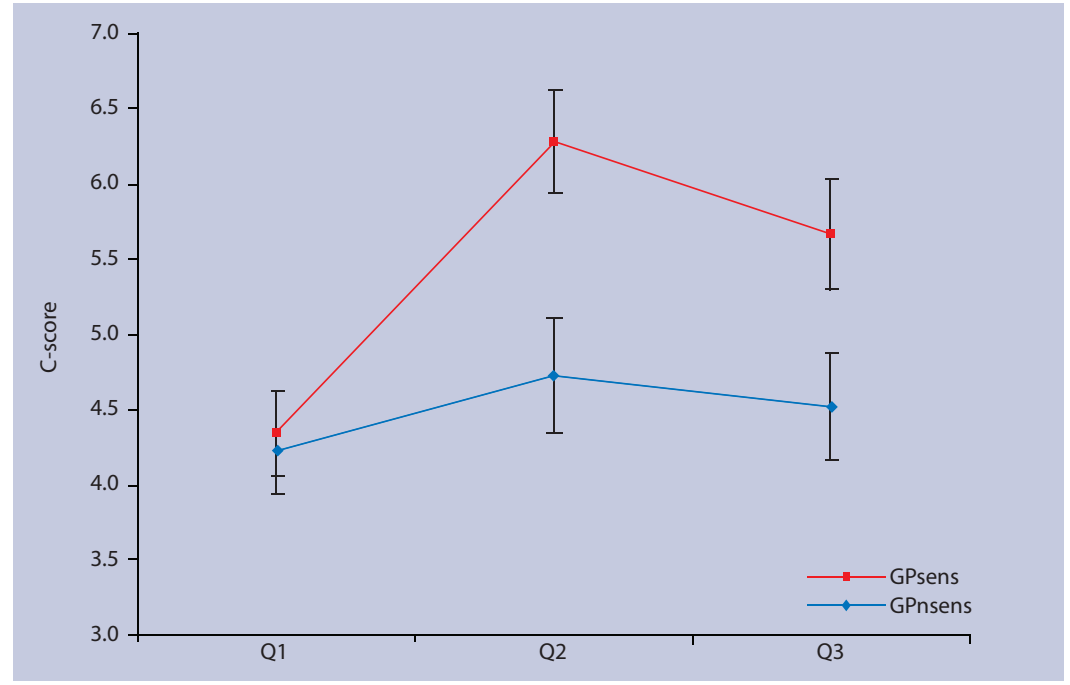

Figure 2. c-scores in GPsens and GPnsens who responded in all surveys Q1 to Q3. Error bars show $95 \% \mathrm{Cls}$.

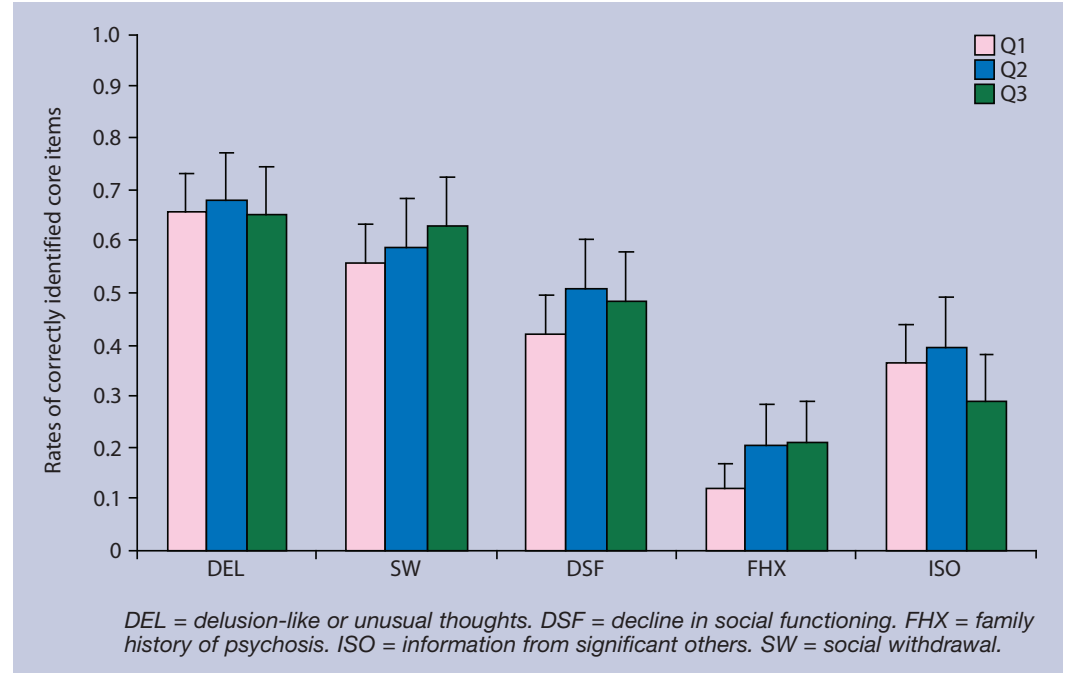

Figure 3. Frequencies of core items identified by GPnsens across surveys Q1 to Q3. Error bars show $95 \%$ Cls.

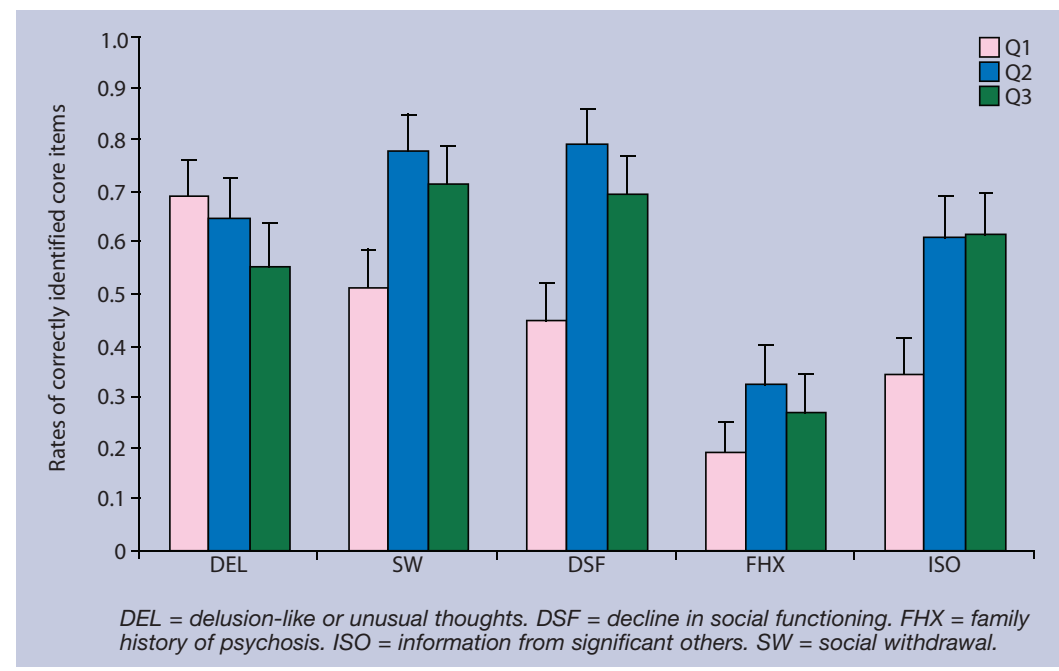

Figure 4. Frequencies of core items identified by GPsens across surveys Q1 to Q3. Error bars show $95 \%$ Cls. 
sensitised GPs showed a highly significant improvement in diagnostic knowledge after a 6month sensitisation period, when compared to their own baseline knowledge scores and when compared to non-sensitised GPs. Moreover, this strong effect was present even after an additional 6month period during which GPs had received no more sensitisation. It is noteworthy that sensitised GPs also improved their knowledge in those particular signs of prodromal schizophrenia that remained under-identified in two previous studies among large samples of GPs, namely social withdrawal and functional decline..$^{13,14}$ The finding that delusion-like ideation was decreasingly indicated by sensitised GPs across the surveys may mirror that the study clinical vignettes, although highlighting all five core items, prioritised awareness of the insidious features of prodromal schizophrenia.

\section{Strengths and limitations of the study}

The main strength of this study lies in its unique position as the first tailored trial of the effect of a repeated vignette-based sensitisation model on GPs' awareness of the warning signs in prodromal schizophrenia. Furthermore, the study evaluated the effect of sensitisation over time. The auto-didactic framework of the sensitisation allows GPs to obtain maximum knowledge in a minimum time. Thus the study model was in keeping with evidence emerging throughout medicine that education for GPs should be multimodal and repetitious. ${ }^{15,16}$ The randomised design and the even distribution of sensitised and non-sensitised GPs at all three survey time points, rules out that the increase in diagnostic knowledge may have been biased by a selection of more interested, and thus more educated, GPs in the field of prodromal schizophrenia.

Study limitations include the response rates, which are often debatable in questionnaire-based postal surveys. However, it is noteworthy that overall two-thirds of all contacted GPs responded at least once to the survey. It is not possible to evaluate whether GPs actively participated at all three sensitisation time points. Thus the degree of significant improvement in knowledge could also have resulted from single and non-repeated sensitisation, which would highlight the value of the applied clinical vignettes. Also, this study, like any research on attitudes, is limited by the tendency that the answers given may not assess actual behaviour, but should be considered more of a 'proxy' measure of intended behaviour. ${ }^{21}$ Along this line, the study did not assess whether the demonstrated increase in diagnostic knowledge in the sensitised GP group was followed by more accurate or increased referrals of patients to secondary care facilities. Compared to telephone or personal interviewing, mail surveys are considered to be inferior in terms of cooperation, interview administration (for example, rapport and confidence), confidentiality, and social desirability. ${ }^{22}$

\section{Comparison with existing literature}

Two recent studies that focused on first-episode psychosis evaluated the effect of GP training on reducing the duration of untreated psychosis and on referral rates to early-intervention services. The Lambeth Early Onset Crisis Assessment Team Study in London included a single short training session on first-episode psychosis for practices that also had direct access to early-intervention services. GPs in the intervention group were more likely to refer their patients to mental health services than GPs in the control group. No difference, however, was found in the duration of untreated psychosis between practices. ${ }^{17}$ The BiRmingham Early Detection In untREated psyChosis Trial (REDIRECT) was conducted in 110 GP practices and included multimodal education. Referral rates to earlyintervention services and duration of untreated psychosis did not differ between the intervention and the non-intervention groups, while training facilitated access to specialist teams. ${ }^{9}$

The present study is the first to dispense fully with educational sessions. At least at some stage, all previous models applied educational programmes for GPs of up to several hours. ${ }^{23-26}$ Overall, such extended programmes to increase awareness of mental disorders have, at best, yielded moderately sustained effects. ${ }^{24-27}$

\section{Implications for clinical practice}

Primary care practitioners appear to be one of the most pivotal professional groups along the helpseeking pathways of patients with prodromal schizophrenia. ${ }^{6-9}$ This trial, and the wider evidence base, ${ }^{28}$ suggest that psychiatrists can substantially support GPs in their difficult task of detecting the early features of this severe mental disease.

\section{Funding body}

The study was supported by an educational grant from the RRMA (Recherche et Réalisation en Médecine Appliquée), Switzerland.

\section{Ethical approval}

The study did not require any ethical approval.

\section{Competing interests}

The authors have stated that there are none; all authors are independent from the funding body and the views expressed in this article have not been influenced by the funding source.

\section{Acknowledgements}

We would like to thank all participating GPs and the committees of the 'Verein Berner Hausärzte', 
'Aerztegesellschaft Basel-Landschaft', and the 'Gesellschaft der Ärzte am Zürichsee'.

\section{Discuss this article}

Contribute and read comments about this article on the Discussion Forum: http://www.rcgp.org.uk/bjgp-discuss

\section{REFERENCES}

1. Marshall M, Lewis S, Lockwood A, et al. Association between duration of untreated psychosis and outcome in cohorts of firstepisode patients: a systematic review. Arch Gen Psychiatry 2005; 62(9): 975-983.

2. Perkins DO, Gu H, Boteva K, Lieberman JA. Relationship between duration of untreated psychosis and outcome in first-episode schizophrenia: a critical review and meta-analysis. Am J Psychiatry 2005; 162(10): 1785-1804.

3. Yung AR, McGorry PD, McFarlane CA, et al. Monitoring and care of young people at incipient risk of psychosis. Schizophr Bull 1996; 22(2): 283-303.

4. Miller TJ, McGlashan TH, Woods SW, et al. Symptom assessment in schizophrenic prodromal states. Psychiatr Q 1999; 70(4): 273-287.

5. Häfner $\mathrm{H}$, Löffler W, Maurer K, et al. Depression, negative symptoms, social stagnation and social decline in the early course of schizophrenia. Acta Psychiatr Scand 1999; 100(2): 105-118.

6. Lincoln CV, Harrigan S, McGorry PD. Understanding the topography of the early psychosis pathways. Br J Psychiatry 1998; 172(Suppl 33): 21-25.

7. Skeate A, Jackson C, Birchwood M, Jones C. Duration of untreated psychosis and pathways to care in first-episode psychosis. Investigation of help-seeking behaviour in primary care. $\mathrm{Br} \mathrm{J}$ Psychiatry 2002; 43(Suppl): 73-77.

8. Platz C, Umbricht DS, Cattapan-Ludewig K, et al. Help-seeking pathways in early psychosis. Soc Psychiatry Psychiatr Epidemiol 2006; 41(12): 967-974.

9. Lester H, Birchwood M, Freemantle N, et al. REDIRECT: cluster randomized controlled trial of GP training in first-episode psychosis. Br J Gen Pract 2009; 59(563): 403-408.

10. Churchill R, Allen J, Denman S, et al. Do the attitudes and beliefs of young teenagers towards general practice influence actual consultation behaviour? Br J Gen Pract 2000; 50(461): 953-957.

11. Biddle L, Donovan JL, Gunnell D, Sharp D. Young adults' perception of GPs as help source for mental distress: a qualitative study. $\mathrm{Br} \mathrm{J} \mathrm{Gen}$ Pract 2006; 56(533): 924-931.

12. Mauerhofer A, Berchtold A, Michaud P-A, Suris J-C. GPs' role in the detection of psychological problems of young people: a populationbased study. Br J Gen Pract 2009; 59(566): 308-314.

13. Simon AE, Lauber C, Ludewig K, et al. General practitioners and schizophrenia: results from a national survey. Br J Psychiatry 2005; 187: 274-281.

14. Simon AE, Lester H, Tait L, et al. The International Study on General Practitioners and Early Psychosis (IGPS). Schizophr Res 2009; 108(1-3): 182-190.

15. Gilbody S, Whitty P, Grimshaw J, Thomas R. Educational and organisational interventions to improve the management of depression in primary care: a systematic review. JAMA 2003; 289(23): 3145-3151.

16. Bloom BS. Effects of continuous medical education on improving physician clinical care and patient health: a review of systematic reviews. Int J Technol Assess Health Care 2005; 21(3): 380-385.

17. Power P, Iacoponi E, Reynolds N, et al. The Lambeth Early Onset Crisis Assessment Team Study: general practitioner education and access to an early detection team in first-episode psychosis. $\mathrm{Br} \mathrm{J}$ Psychiatry 2007; 51(Suppl): 133-139.

18. Birchwood M, Todd P, Jackson C. Early Intervention in psychosis. $\mathrm{Br}$ J Psychiatry 1998; 172: 53-59.

19. Cannon T, Cadenhead K, Cornblatt B, et al. Prediction of psychosis in youth at high clinical risk. A multiside study in North America. Arch Gen Psychiatry 2008: 65(1): 28-37.

20. Kety SS. The significance of genetic factors in the etiology of schizophrenia: results from the national study of adoptees in Denmark. J Psychiatr Res 1987; 21(4): 423-429.

21. Penn DL, Corrigan PW. The effects of stereotype suppression on psychiatric stigma. Schizophr Res 2002; 55(3): 269-276.

22. Fowler FJ Jr. Survey research methods. 3rd edn. Thousand Oaks, CA: Sage Publications, Inc, 2002.

23. Van Os TW, Ormel J, van den Brink RH, et al. Training primary care physicians improves the management of depression. Gen Hosp Psychiatry 1999; 21(3): 168-176.

24. Tiemens BG, Ormel J, Jenner JA, et al. Training primary care physicians to recognize, diagnose and manage depression: does it improve patient outcomes? Psychol Med 1999; 29(4): 833-845.

25. Worrall G, Angel J. Effectiveness of an educational strategy to improve family physicians' detection and management of depression: a randomized controlled trial. CMAJ 1999; 161(1):37-40.

26. Croudace T, Evans J, Harrison G, et al. Impact of the ICD-10 Primary Health Care (PHC) diagnostic and management guidelines for mental disorders on detection and outcome in primary care. $\mathrm{Br} \mathrm{J}$ Psychiatry 2003; 182: 20-30.

27. Kendrick T. Why can't GPs follow guidelines on depression? BMJ 2000; 320(7229): 200-201.

28. Oud MJ, Schuling J, Slooff CJ, Meyboom-de Jong B. How do general practitioners experience providing care for their psychotic patients? BMC Fam Pract 2007; 8: 37 . 


\section{Appendix 1. Example of clinical vignette presented to GPs. ${ }^{a}$}

\section{Clinical vignette}

A mother makes an appointment with you for her son. You have known the mother for years. She tells you that her son has been staying home for the past 4 months instead of going to work. He is 18 years old and in the third year of his apprenticeship. In addition, it is several months since he last went to training with his football club. He has become 'somehow different'. When making the appointment, the mother asks you whether her son may be suffering from some physical illness and asks you to examine him. During the first appointment, at which the mother is not present, on being asked how he feels, the son tells you that everything is actually fine, that he feels tired now and then, and that it is his mother who is exaggerating.

\section{Comments}

The mother is in fact providing you with valuable information: a social withdrawal has been going on for months, accompanied by loss of motivation, as well as a decline in social functioning, which has also lasted for months - both of these points being among the most important indications that you could obtain. The information obtained from relatives is of such importance because the patients themselves, as with patient in this case, often deny having any problems. The mother experiences her son as 'changed'. Furthermore, as you have known the family for years, you also know whether there is a history of psychosis in the family, a fact that would increase the risk. While talking to the patient, try to find out if the way of reasoning of the patient is unusual.

Of the following 20 indicators of prodromal schizophrenia, highlight the 5 items that are the most important indicators.

- hallucinations

delusion-like or unusual thoughts

social withdrawal

- psychosomatic symptoms

- suicidality

- depression/anxiety

- bizarre behaviour

- substance abuse

- conflicts with parents/teachers/employers

decline in social functioning (school/work)

- personal history

$\checkmark$ family history

information from significant others (family/employer/teacher)

- neurological examination

- neuropsychological examination

- other examinations (for example, X-ray or EEG)

- laboratory examinations

- urine toxicology

- counselling with psychiatrist

- other: 